\title{
Visualization of Cell Migration During Histogenesis in Living Chick Retina Slices
}

\author{
Joel B. Sheffield*, Cheung Law*, Man-Sang Hahn*, M.W. Davidson ${ }^{* *}$ \\ *Department of Biology, Temple University, Philadelphia, PA, 19122 \\ **National High Magnetic Field Laboratory, Florida State University, Tallahassee, FL 32310
}

The cells of the retina are arranged in specific layers that arise during development. These layers are stratified both as to structure and to function. The original tissue that gives rise to the retina is, however, a single cell epithelium. One of the central problems in the development of the retina is to understand the processes that give rise to the layers.

In initial studies, we used DIC and Hoffman optics to visualize the cell migration patterns in living slices of developing chick retinas using optical sectioning and time-lapse microscopy. Chick retinas of embryonic ages from 6 to 18 days of incubation were dissected in cold Tyrode's solution and embedded in $8 \%$ low gelation temperature agarose (Sigma type IX-A) in Tyrode's solution. The embedded tissue was kept cold on a chilled surface. The tissue was sliced through the apical/basal axis of the retina with a Smith-Farquhar Tissue Slicer, into sections of 125 to 150 microns thickness. The slices were transferred to a thermally regulated chamber containing $1 \mathrm{ml}$ of Ham's F-12 medium supplemented with fetal calf serum to $10 \%$. The $\mathrm{pH}$ of the medium was maintained at 7.6, and. the temperature at $37^{\circ} \mathrm{C}$. In the youngest retinas examined, many cells were seen to migrate within the tissue constrained to the apical/basal axis of the tissue. They move in one direction, reverse, and then resume their original direction. The cells achieved maximum speeds of 10 microns/minute, although there was considerable variation in rate of movement even for any one cell. In older retinas, of 8-15 days of incubation, overall cellular migration was reduced, although there was continuing intercellular activity. Individual small structures (either cells or nuclei within cell bodies) migrated from the apical or basal portion of the tissue to positions within the growing inner nuclear layer, and stopped. This is followed by a period of little cellular migration, during which time the plexiform layers expand. Late in development, we observed lateral migration of cells through the inner plexiform layer of E18 retinas (Figure 1d). There was no significant lateral movement beyond approximately one cell diameter, except in the late retinas.

In order to obtain additional information about the topography of this process, intact retinas of different embryonic ages were exposed to a solution of rhodamine B-conjugated dextran 10,000 (Molecular Probes cat \# D1824) in culture medium for one half hour before slicing the tissue. The slices were then placed into a temperature controlled slide chamber and examined with a Leica SP1 confocal microscope at four depths within the tissue, using a long working distance 40x objective. Images for time-lapse analysis were obtained every twenty seconds, using low levels of illumination, and $256 \times 256$ scans.

Cells with processes at the surfaces of the tissue took up the stain, and were easily visualized, extending from the apical to the basal surfaces of the tissue (Figure 2). In younger retinas, we observed occasional extension and withdrawal of processes by these cells, but in most cases, they remained still, even though unlabeled cells, visualized by transmission DIC were moving. The labeled cells appear to be the matrix on which the other cells migrate, assuming the role of radial glia in other neural systems. 

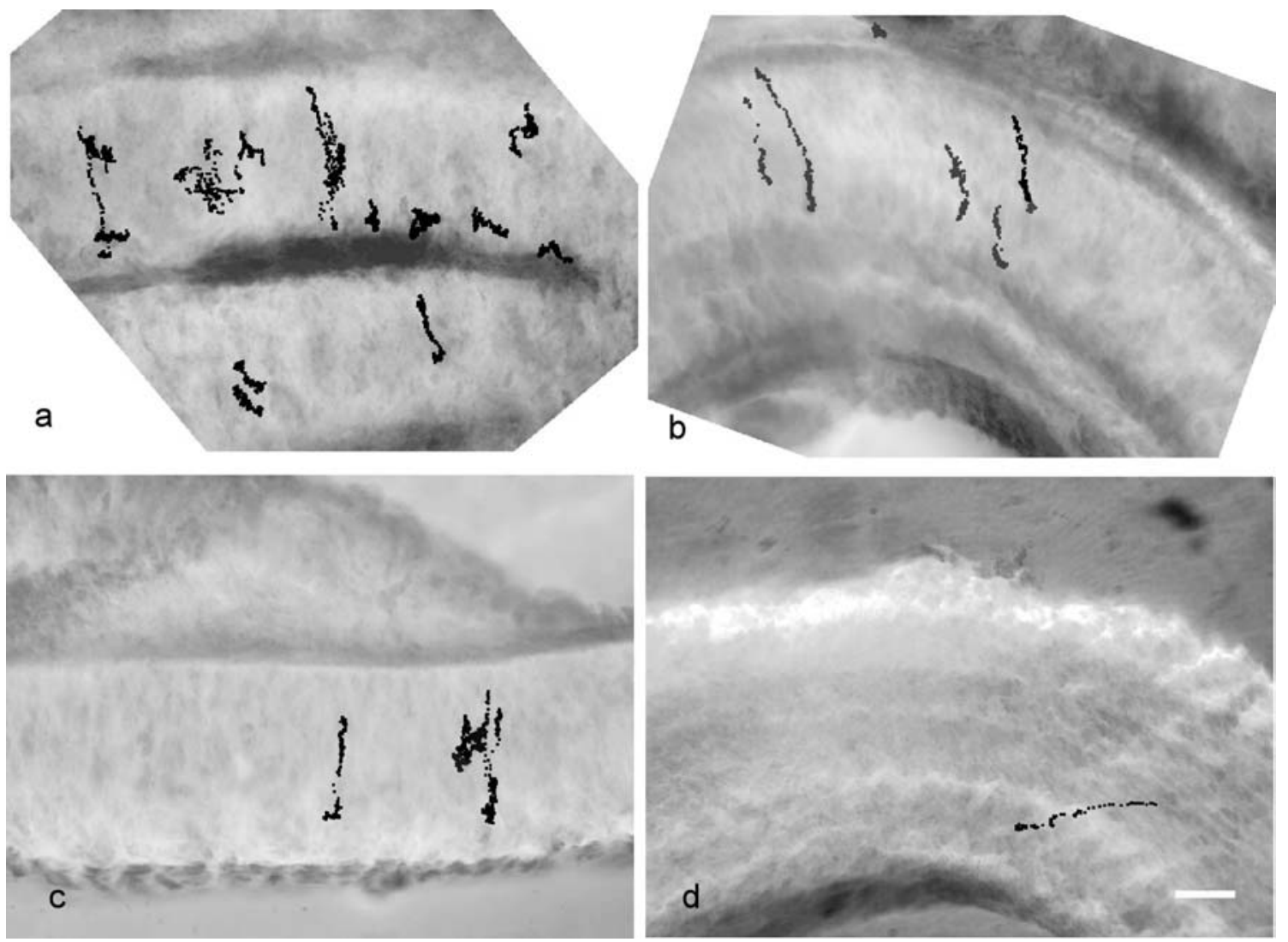

Figure 1. Projection images of selected cells in time-lapse sequences of four embryonic retinal ages. Individual cells were marked in each image in the series, and then the stack of approximately 250 images was compressed into a single image. The resulting image superimposes the marks for each cell throughout the series on an averaged image of the tissue. The slight lateral movement of the paths is due to drifting of the tissue. a. Six day retina. b. Eight day retina. c. Nine day retina. d. Eighteen Day retina. The bar represents 20 micrometers.
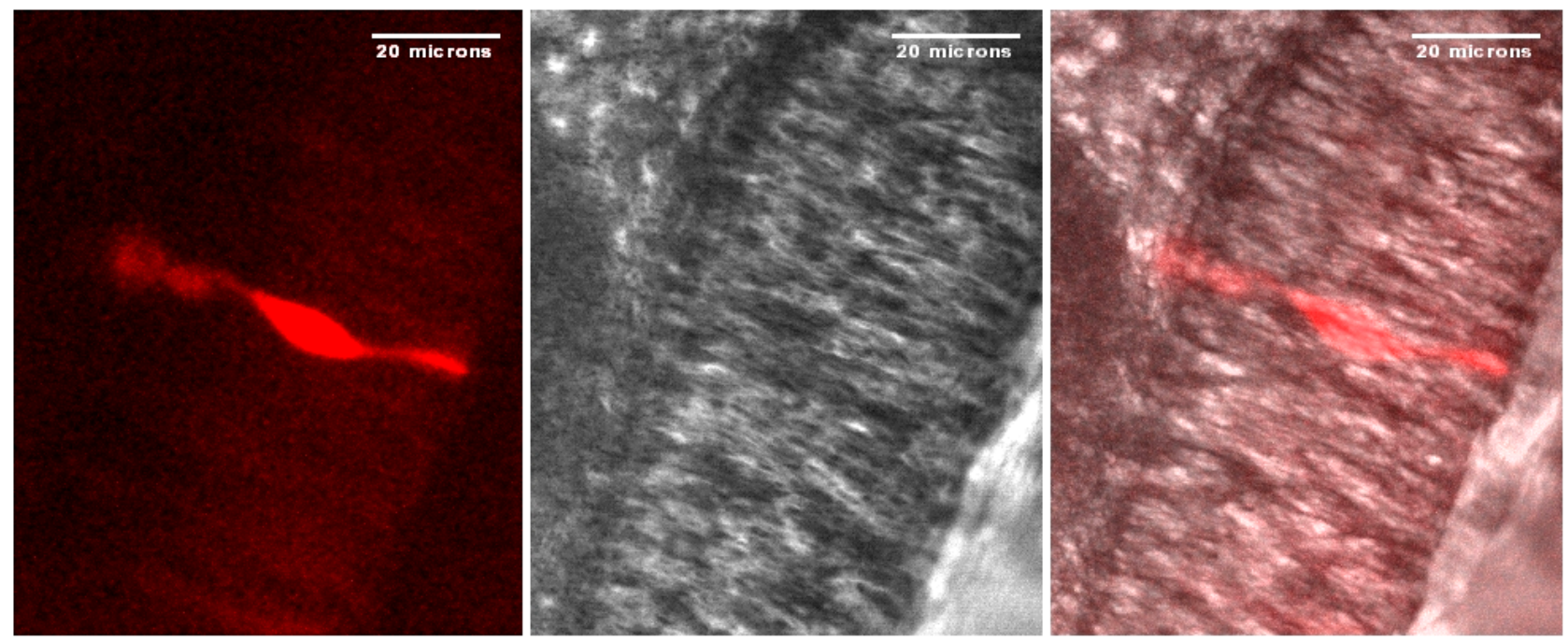

Figure 2. Z-Projection of four confocal slices of a four day embryonic retina exposed to rhodaminedextran for one hour prior to observation. The basal (inner) surface of the retina is on the right. The bar represents 20 micrometers. 